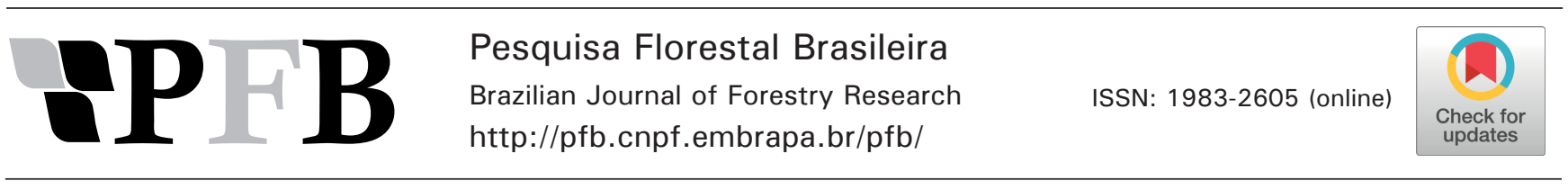

\title{
Respostas fisiológicas de plantas jovens de macaúba a condições de seca cíclica
}

\author{
Clenilso Sehnen Mota ${ }^{1 *}$, Marco Antonio Oliva Cano² \\ ${ }^{1}$ Instituto Federal Catarinense, Estrada do Redentor, 5.665, CEP 89163-356, Rio do Sul, SC, Brasil \\ ${ }^{2}$ Universidade Federal de Viçosa, Av. Peter Henry Rolfs, s/n, CEP 36570-900, Viçosa, MG, Brasil
}

"Autor correspondente:

csm.sehnen@gmail.com

Termos para indexação:

Acrocomia aculeata

Balanço energético

Osmólitos

Index terms:

Acrocomia aculeata

Energy balance

Osmolytes

Histórico do artigo:

Recebido em 01/10/2015

Aprovado em 11/08/2016

Publicado em 30/09/2016

doi: $10.4336 / 2016 . p f b .36 .87 .1061$
Resumo - O objetivo do presente estudo foi avaliar os impactos de três ciclos de seca/ reirrigação sobre os rendimentos quânticos da fluorescência da clorofila $a$, acúmulo de prolina e ajustamento osmótico. Dez plantas cultivadas em vasos com capacidade de $150 \mathrm{~L}$ de solo foram submetidas a três ciclos sucessivos de seca/reirrigação $(\mathrm{S} / \mathrm{R})$ e outras dez foram mantidas irrigadas em capacidade de campo. Os ciclos $S / R$ não promoveram ajustamento osmótico nas plantas mantidas com suspensão da irrigação (CSI), apesar do acúmulo de prolina. A fluorescência mínima adaptada ao escuro aumentou e a eficiência quântica máxima do PSII teve redução nas plantas CSI. O rendimento quântico de conversão de energia fotoquímica do fotossistema II (PSII) e a taxa aparente de transporte de elétrons apresentaram redução, enquanto o rendimento quântico de dissipação regulada de energia não fotoquímica no PSII apresentou aumento nas plantas do grupo CSI em relação às plantas sem suspensão da irrigação (SSI). O rendimento quântico de dissipação não regulada de energia não fotoquímica do PSII e os teores de clorofilas e carotenoides não apresentaram alterações entre as plantas CSI e SSI ao longo dos ciclos S/R. Observa-se, assim, que a macaúba apresenta um eficiente mecanismo de dissipação de energia não fotoquímica.

\section{Physiological responses of macaúba young plants to cyclical drought conditions}

\begin{abstract}
The objective of this study was to evaluate the impact of three cycles of drought/irrigation on the quantum yields of chlorophyll a fluorescence, proline accumulation and osmotic adjustment. Ten plants were submitted to three consecutive cycles of drought/irrigation (D/I) and the other ten were maintained at field capacity. Cycles D/I did not promote osmotic adjustment in plants with suspension of irrigation (WSI), despite the proline accumulation. The minimum fluorescence adapted to dark increased and the maximum quantum efficiency of PSII was reduced in WSI plants. Quantum yield of photochemical energy conversion of photosystem II (PSII) and the apparent rate of electron transport decreased, while quantum yield dissipation regulated non-photochemical energy in PSII showed an increase in WSI plants over those without suspension of irrigation (WOSI). Quantum yield of no regulated dissipation of PSII non-photochemical energy and chlorophyll and carotenoid content showed no change between WSI and WOSI plants over D/I cycles. Thus it was observed that macaubeira presents an efficient mechanism of non-photochemical energy dissipation.
\end{abstract}

\section{Introdução}

A seca é um estresse multidimensional que afeta as plantas nos vários níveis de organização, desde células até a planta inteira. O déficit hídrico causado pela seca induz as plantas a diferentes respostas bioquímicas e fisiológicas, representando o fator ambiental que mais influência seu crescimento e produção, em escala mundial (Claeys \& Inzé, 2013; Lipiec et al., 2013). O fechamento estomático é um dos primeiros processos 
fisiológicos a ser afetado pela seca em plantas, sendo a fotossíntese afetada diretamente (Chaves et al., 2009). O fechamento estomático é muitas vezes considerado uma resposta fisiológica antecipada à deficiência hídrica, que resulta em diminuição da fotossíntese através da limitação da entrada de $\mathrm{CO}_{2}$ no mesófilo (Cornic, 2000).

A capacidade das plantas de recuperarem a capacidade fotossintética após a irrigação precedida por um período de seca remete à capacidade da planta de evitar e/ou suportar um estresse hídrico e ditará a resistência à seca, além de impedir decréscimo na produtividade da cultura (Chaves et al., 2009). A análise da recuperação de diferentes componentes fotossintéticos após a reidratação a partir de diferentes intensidades de estresses hídricos é de grande importância para o entendimento dos processos fisiológicos que ocorrem durante a reidratação das plantas (Flexas et al., 2006).

Danos ao fotossistema II (PSII) são relatados em condições de deficiência hídrica (Calatayud et al., 2006), bem como alterações no uso e dissipação da energia absorvida, como relatado por Suresh et al. (2010) em plantas de Elaeis guineenses Jacq. Em Cocos nucifera L. (Gomes et al., 2008) e Elaeis guineenses Jacq. (Cha-Um et al., 2012; Suresh et al., 2012), o aproveitamento energético representado pela eficiência quântica máxima do PSII $\left(\mathrm{F}_{\mathrm{v}} / \mathrm{F}_{\mathrm{m}}\right)$ e o rendimento quântico de conversão de energia fotoquímica do PSII ( $\phi$ PSII) são afetados, principalmente este último, sob deficiência hídrica. Nessas condições, a diminuição de assimilação liquida de $\mathrm{CO}_{2}(A)$ pode levar à redução do $\phi$ PSII; consequentemente, a energia absorvida que seria utilizada para $A$ será dissipada, pelo aumento da emissão de fluorescência da clorofila $a$ e na forma de calor, representado principalmente pelo coeficiente de dissipação não fotoquímica (Calatayud et al., 2006). Todavia, o funcionamento do ciclo água-água e a fotorrespiração em condições de deficiência hídrica moderada podem manter a taxa de transporte de elétrons (ETR) e o $\phi$ PSII (Suresh et al., 2012).

A tolerância das plantas à seca pode se dar, ainda, pelo acúmulo de osmólitos compatíveis, como açúcares, aminoácidos, álcoois e amônias quaternárias, sendo essa uma característica importante para a superação de estresses ambientais. Essas moléculas atuam na redução da atividade da água e redução do potencial osmótico da célula, para manter a turgidez da mesma e manter o crescimento e sobrevivência. As moléculas osmolíticas podem atuar também como osmoprotetores, como a prolina, que estabiliza estruturas macromoleculares e membranas subcelulares (Krasensky \& Jonak, 2012). Em Euterpe oleracea Mart. (Calbo \& Moraes) e C. nucifera (Gomes et al., 2008) foi identificado um ajustamento osmótico em resposta à seca, porém sem a identificação da molécula que foi a responsável pelo ajustamento.

Experimentos relacionados à seca são conduzidos frequentemente em pequenos vasos, impondo-se déficits hídricos de maneira relativamente rápida, não permitindo que as plantas se aclimatem à seca. Poucos são os estudos com seca que envolvem ciclos repetitivos de seca e posterior irrigação, buscando simular períodos reais de estiagem e posterior precipitação pluviométrica em condições bem próximas da realidade.

Esse trabalho tem por hipótese que macaubeira jovens apresentam ajustamentos osmóticos, aumento na síntese de prolina, aclimatação da fase fotoquímica da fotossíntese e eficiente regulação no balanço de energia, quando submetidas à deficiência hídrica cíclica, em simulação a condições reais de seca. Este trabalho objetivou avaliar o impacto de três ciclos repetitivos de seca/reirrigação sobre os rendimentos quânticos da fluorescência da clorofila $a$, ajustamento osmótico e acúmulo de prolina em plantas jovens de macaúba.

\section{Material e métodos}

\section{Condições de cultivo e tratamentos}

$\mathrm{O}$ experimento foi conduzido em casa de vegetação (2045'26'S, 42 52'22”W). As mudas utilizadas de macaúba (Acrocomia aculeata (Jacq.) Lodd. ex Mart., estavam com dez meses de idade quando recebidas do viveiro comercial. As sementes utilizadas para a produção das mudas são procedentes do município de Florestal, MG (1953'10”'S, 44²4'51’W).

Os vasos de cultivo das plantas no experimento apresentavam capacidade de $150 \mathrm{~L}$ e formato cônico, com diâmetro superior, inferior e altura de $0,7,0,5$ e 0,5 $\mathrm{m}$, respectivamente. $\mathrm{O}$ substrato utilizado foi composto de uma mistura de solo, areia e esterco bovino curtido na proporção de 3:1:1 (v:v:v), sendo as plantas cultivadas nessas condições por nove meses. Foram feitas correções de fertilidade do substrato e adubação de manutenção, de acordo com Pimentel et al. (2011). A irrigação das plantas foi realizada diariamente, a fim de evitar possíveis deficiências hídricas.

As variações climáticas de temperatura e a radiação solar no interior da casa de vegetação foram quantificadas 
com um termistor não linear e um piranômetro LI-200SA, respectivamente, ambos acoplados a um datalogger LI-1200 Data Set Recorder (LI-COR, Biosciences, USA). A umidade relativa do ar foi medida com um termohigrógrafo, calibrado com um psicrômetro de bulbo úmido ventilado.

Aos 19 meses de idade, as mudas de macaúba foram submetidas à suspensão da irrigação até que a assimilação liquida de $\mathrm{CO}_{2}(A)$ fosse próximo a zero, sendo esse momento considerado o ponto de máximo estresse (PME). Nesse momento, a hidratação foi retomada até que a recuperação de $A$ fosse superior $85 \%$, em relação às plantas sem a suspenção da irrigação (SSI). Esse procedimento foi repetido duas vezes, perfazendo um total de três ciclos de seca/reirrigação $(\mathrm{S} / \mathrm{R})$, simulando assim uma seca em condições naturais. As medições fisiológicas foram realizadas em intervalos de aproximadamente 10 dias, em folíolos da região mediana da segunda folha completamente expandida.

\section{Trocas gasosas}

A assimilação liquida de $\mathrm{CO}_{2}$ foi avaliada entre as $8 \mathrm{e}$ $11 \mathrm{~h}$, com um sistema portátil de medição de fotossíntese LI-6400 (LI-COR Biosciences, USA). O equipamento também forneceu a condutância estomática $\left(g_{s}\right)$ e a relação entre a concentração atmosférica e a intercelular de $\mathrm{CO}_{2}(\mathrm{Ci} / \mathrm{Ca})$. A radiação fotossinteticamente ativa foi fornecida por uma fonte luminosa artificial (azul/ vermelho, modelo 6400-02B, Li-Cor Biosciences, USA), sendo fixada em $1500 \mu \mathrm{mol} \mathrm{m} \mathrm{m}^{-2} \mathrm{~s}^{-1}$. A concentração atmosférica de $\mathrm{CO}_{2}$ oscilou de 380 a $400 \mu \mathrm{mol} \mathrm{mol}^{-1}$, durante as leituras. As leituras foram registradas pelo equipamento quando o coeficiente de variação foi inferior a $0,5 \%$.

\section{Potencial hídrico, osmótico e ajustamento osmótico}

O potencial hídrico foliar na antemanhã $\left(\Psi_{\mathrm{am}}\right)$ foi avaliado às $4 \mathrm{~h} 30 \mathrm{~min}$ em um folíolo por planta, com uma câmara de pressão tipo Scholander (modelo Skye SKPM 1400, Skye Instruments LTD., UK). As medições foram realizadas em folíolos o mais próximo possível do folíolo utilizado para as avaliações de trocas gasosas. A pressão do interior da câmara de pressão foi aumentada lentamente, a uma taxa de $0,015 \mathrm{MPa} \mathrm{s}^{-1}$, com nitrogênio comprimido.

Para determinação do potencial osmótico, amostras de folíolos foram coletadas e armazenadas a $-20{ }^{\circ} \mathrm{C}$, sendo posteriormente descongeladas e prensadas através de prensa manual. O conteúdo celular extravasado foi utilizado para determinação de potencial osmótico $\left(\Psi_{\mathrm{S}}\right)$, em número de cinco repetições por nível de irrigação. Devido à baixa quantidade de conteúdo celular obtido, foi feita uma diluição de quatro vezes, em todas as amostras. A determinação do $\Psi_{\mathrm{S}}$ foi realizada pelo método crioscópico, usando um crioscópio eletrônico microprocessado (modelo MK 540, ITR Instrumentos Ltda., Brasil). Os valores de graus Hortvet $\left({ }^{\circ} \mathrm{H}\right)$ fornecidos pelo crioscópio foram transformados para graus Celsius $\left({ }^{\circ} \mathrm{C}\right)$ (equação 1), segundo Baer \& Baldwin (1984), a transformação de ${ }^{\circ} \mathrm{C}$ para Osmol foi feita segundo Henriques \& Rosado (1999), pela equação 2, onde, 1,86 é o ponto de congelamento de $1 \mathrm{~mol}$ de qualquer soluto. Considerando que 1 Osmol equivale a -2,271 $\mathrm{MPa}$, a transformação de Osmol para $\mathrm{MPa}$ foi obtida pela equação 3. Para evitar efeitos passivos associados à perda de água, os valores de $\Psi_{\mathrm{S}}$ (sem e com suspenção da hidratação) foram multiplicados por um fator de correção $(F C)$ obtido pela equação 4 .

$$
\begin{aligned}
& { }^{\circ} \mathrm{C}=\frac{\left(0,1915 *{ }^{\circ} \mathrm{H}\right)-(-0,0004785)}{0,199} \\
& \text { Osmol }=\frac{-{ }^{\circ} \mathrm{C}}{1,86} \\
& M P a=-2,271 * \text { Osmol } \\
& F C=\frac{M F-M S}{M S *[1 / 0,3-1]}
\end{aligned}
$$

onde, $\mathrm{MPa}=$ Unidade de Pressão, Osmol = Unidade de osmolaridade, $\mathrm{FC}=$ fator de correção, e $M F$ e $M S=$ massa fresca e seca foliar, respectivamente.

O FC é baseado na massa seca dos tecidos, que retorna a um potencial osmótico em um teor de água comum de $70 \%$ da massa fresca, para os dois tratamentos (Alves \& Setter, 2004). O ajustamento osmótico (AO) foi calculado pela diferença de potencial osmótico corrigido $\left(\Psi_{\mathrm{SC}}\right)$ entre as plantas sem e com suspenção da hidratação.

\section{Fluorescência da clorofila $a$}

As variáveis de fluorescência da clorofila $a$, foram obtidas com o uso de um fluorômetro modulado MiniPAM (Walz, Effeltrich, Alemanha). O rendimento quântico máximo do PSII $\left(\mathrm{F}_{\mathrm{v}} / \mathrm{F}_{\mathrm{m}}\right)$ foi calculado a partir dos chamados parâmetros de escuro, pela equação 5 . 


$$
F_{v} / F_{m}=\frac{\left(F_{m}-F_{0}\right)}{F_{m}}
$$

em que $\mathrm{F}_{0}$ e $\mathrm{F}_{\mathrm{m}}$ são a fluorescência mínima e máxima, respectivamente, do tecido vegetal adaptado ao escuro, obtidos na antemanhã. $\mathrm{F}_{0}$ foi obtido a partir da iluminação do tecido vegetal por uma luz vermelha modulada de baixa intensidade $\left(0,03 \mu \mathrm{mol} \mathrm{m}^{-2} \mathrm{~s}^{-1}\right)$, e $\mathrm{F}_{\mathrm{m}}$, por um pulso de luz saturante $\left(6.000 \mu \mathrm{mol} \mathrm{m}^{-2} \mathrm{~s}^{-1}\right) \mathrm{com}$ duração de $0,8 \mathrm{~s}$.

O rendimento quântico máximo efetivo do PSII ( $\phi$ PSII) foi obtido a partir da equação 6 , segundo Hendrickson et al. (2004).

$$
\phi P S I I=\frac{\left(F_{m}{ }^{\prime}-F\right)}{F_{m}{ }^{\prime}}
$$

em que $F_{m}$ ' e $F$ são a fluorescência máxima e a fluorescência variável, respectivamente, quando o tecido vegetal está sob luz actínica com intensidade de 1.500 $\mu \mathrm{mol} \mathrm{m} \mathrm{m}^{-2} \mathrm{~s}^{-1}$ com duração de $50 \mathrm{~s}$.

O rendimento quântico da dissipação regulada de energia não fotoquímica no PSII ( $\phi \mathrm{NPQ})$ e o rendimento quântico de dissipação não regulada de energia não fotoquímica do PSII ( $\phi \mathrm{NO})$ foram obtidos segundo Hendrickson et al. (2004), conforme as equações 7 e 8 , respectivamente.

$$
\begin{aligned}
& \phi N P Q=\frac{F}{F_{m}{ }^{\prime}}-\frac{F}{F_{m}} \\
& \phi N O=\frac{F}{F_{m}}
\end{aligned}
$$

onde: $\mathrm{F}_{\mathrm{m}}$ ' e $\mathrm{F}$ são a fluorescência máxima e a fluorescência variável, respectivamente, quando o tecido vegetal está sob luz e, $\mathrm{F}_{\mathrm{m}}$ é a fluorescência máxima, do tecido vegetal adaptado ao escuro.

A taxa aparente de transporte de elétrons do PSII (ETR) foi obtida pela equação 9 .

$$
E T R=\phi P S I I \times R F A \times a \times 0,5
$$

em que $\phi$ PSII é o rendimento quântico efetivo do PSII; $a$ é a fração incidente de RFA, que é absorvida pelo tecido foliar, considerando-se um valor de 0,84 ; e o fator 0,5 é o valor que corresponde à fração da energia de excitação distribuída igualmente entre o PSII e PSI.

\section{Analises bioquímicas}

Os teores de pigmentos fotossintéticos por unidade de área foliar foram determinados em extratos obtidos a partir de discos foliares com dimetilsulfóxido (DMSO). Foram coletados dois discos foliares $\left(77 \mathrm{~mm}^{2}\right)$ por repetição, os quais foram incubados em $5 \mathrm{~mL}$ de DMSO (saturado com $\mathrm{CaCO}_{3}$ ) a $65{ }^{\circ} \mathrm{C}$ por $36 \mathrm{~h}$; em seguida, procedeu-se à leitura de absorbância dos extratos em espectrofotômetro. Os teores de clorofilas e carotenoides foram calculados segundo Wellburn (1994).

Para análise de prolina, amostras de tecido vegetal foram coletadas na antemanhã e imediatamente congeladas em nitrogênio líquido e armazenadas a $-80{ }^{\circ} \mathrm{C}$. Posteriormente, foram liofilizadas a $-60{ }^{\circ} \mathrm{C}$ e armazenadas a $-20{ }^{\circ} \mathrm{C}$, até o momento da análise. A concentração de prolina foi determinada pelo método de ninhidrina, segundo Bates et al. (1973).

\section{Potencial matricial no substrato de cultivo}

Em amostras de substratos coletadas na profundidade de $0,4 \mathrm{~m}$, foi quantificada a umidade gravimétrica, com secagem a $105^{\circ} \mathrm{C}$ até massa constante. Para construção da curva característica de retenção de água no solo, amostras do substrato foram saturadas com água e submetidas a diferentes pressões em câmara de Richards (Richards, 1948). Para a obtenção do potencial mátrico nas amostras do substrato, foram utilizados os valores de umidade gravimétrica e a equação de van Genuchten, ajustada aos pontos da curva característica de retenção de água no solo pelo software SWRC (Dourado-Neto et al., 2000).

\section{Delineamento experimental e analise estatística}

As plantas foram distribuídas em delineamento inteiramente casualizado, com dez repetições em cada tratamento (sem e com suspenção de irrigação). Para as análises de prolina e potencial osmótico, foram aleatorizadas cinco plantas para as análises. Os teores de prolina, pigmentos fotossintéticos e a fluorescência da clorofila foram avaliados em intervalos de aproximadamente 10 dias. As demais características foram avaliadas no PME. Os dados de todas as variáveis foram submetidos à análise de variância, e o teste de Tukey a 5\% de significância foi usado para discriminar as diferenças mínimas significativas entre as médias, quando aplicável. Os procedimentos estatísticos foram realizados utilizando o programa computacional Statistical Analysis System 9.0 (SAS Institute, 2004). 


\section{Resultados e discussão}

O segundo ciclo de seca/reirrigação (S/R), apresentou a maior temperatura média do ar e radiação solar em relação aos demais (Tabela 1). Demonstra-se assim que o segundo ciclo foi o mais adverso, seguido pelo primeiro, sendo o terceiro o mais ameno. Os ciclos apresentaram redução em sua duração com o avançar dos ciclos, apresentando duração de 60,41 e 38 dias para os ciclos 1, 2 e 3, respectivamente. Gomes et al. (2008), ao simularem ciclo de seca e recuperação em Cocus nucifera, obtiveram variação de duração de 35 a 44 dias para os ciclos.

O potencial mátrico do substrato de cultivo das plantas com suspensão de irrigação (CSI) não pôde ser estimado, pois ficou aquém dos limites da metodologia utilizada, estando abaixo de $-1,5 \mathrm{MPa}$, nos pontos de máximo estresse (PME). Todavia, após a reirrigação, as plantas CSI foram capazes de recuperar a turgidez, apresentando potencial hídrico foliar na antemanhã $\left(\Psi_{\mathrm{am}}\right)$ de -0,21 MPa, frente a -1,91 MPa das mesmas no PME, na média dos três ciclos, não diferindo entre os ciclos S/R. Recuperação similar foi observado por Gomes et al.
(2008) em C. nucifera submetidas a ciclos S/R. Gomes et al. (2010) relataram $\Psi_{\text {am }}$ de -1,2 MPa no PME.

Tabela 1. Temperatura média do ar (T), radiação solar (RS) e umidade relativa (UR) no interior da casa de vegetação, e duração dos ciclos de seca/reirrigação (S/R).

\begin{tabular}{|c|c|c|c|c|}
\hline Ciclos & S/R (Dias) & $\mathrm{T}\left({ }^{\circ} \mathrm{C}\right)$ & $\operatorname{RS}\left(\mathbf{M J} \mathbf{m}^{-2} \mathbf{d}^{-1}\right)$ & $\begin{array}{l}\text { UR } \\
(\%)\end{array}$ \\
\hline 1 & 60 & $23,5 b$ & $12 b$ & $80 \mathrm{a}$ \\
\hline 2 & 41 & $25,2 \mathrm{a}$ & $15 \mathrm{a}$ & $77 \mathrm{a}$ \\
\hline 3 & 38 & $23,1 \mathrm{~b}$ & $10 \mathrm{c}$ & $80 \mathrm{a}$ \\
\hline
\end{tabular}

Médias seguidas da mesma letra não diferem entre si pelo teste de Tukey $(\mathrm{p}<0,05)$.

Nos PME, quando a taxa de assimilação liquida de $\mathrm{CO}_{2}(A)$ chegou próximo a zero nas plantas com suspenção da irrigação, os valores de condutância estomática $\left(g_{s}\right)$ e a transpiração $(E)$ foram igualmente insignificantes nas plantas CSI, apresentando 3,7 e 4,9\%, respectivamente, em relação às plantas sem suspensão de irrigação (SSI) (Figura 1). Todavia, houve recuperação acima de $85 \%$ destas após a reirrigação, e acima de $90 \%$ para $g_{s}$ e $E$ nos ciclos 2 e 3 . Recuperação similar de $A, E$ e $g$, foi observado por Gomes et al. (2010) em C. nucifera, e Suresh et al. (2010) em Elaeis guineenses Jacq, submetidas a ciclos $\mathrm{S} / \mathrm{R}$.
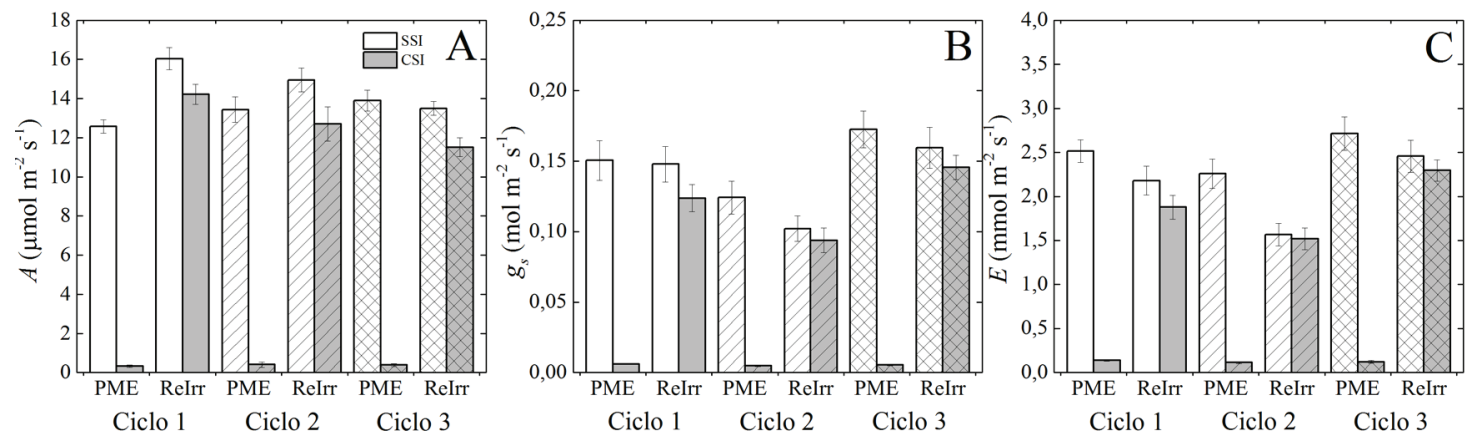

Figura 1. A) Assimilação liquida de $\mathrm{CO}_{2}(A)$; B) condutância estomática $\left(g_{s}\right)$ e C) transpiração $(E)$ em plantas jovens de macaúba, sem (SSI) e com (CSI) suspenção da irrigação, nos ponto de máximo estresse (PME) e na reirrigação (ReIrr), nos três ciclos de seca/reirrigação (ciclo 1 = sem linhas; ciclo $2=$ linha simples; ciclo 3 = linhas cruzadas). As barras indicam os erros-padrão das médias.

O potencial osmótico corrigido $\left(\Psi_{\mathrm{SC}}\right)$, ou seja, após a aplicação do fator de correção (Alves \& Setter, 2004), não diferiu entre as plantas com e sem suspenção da irrigação nos PME em cada um dos ciclos (Figura 2A). Por outro lado, tanto as plantas SSI como as CSI apresentaram valores de $\Psi_{\mathrm{SC}}$ menores no primeiro ciclo, em relação aos demais. O ajustamento osmótico para a macaúba $(0,01-0,02 \mathrm{MPa})$ é muito inferior ao encontrado para outras espécies de palmeiras, como açaí, 0,36 MPa (Calbo \& Moraes, 2000), e coqueiroanão, 0,05 0,24 MPa (Gomes et al., 2008). Demostrase com isso que a macaubeira não possui capacidade de ajustamento osmótico, como a síntese de açúcares, álcoois, aminoácidos e amônias quartanárias, ou mesmo acúmulos de íons com propriedades osmóticas. 


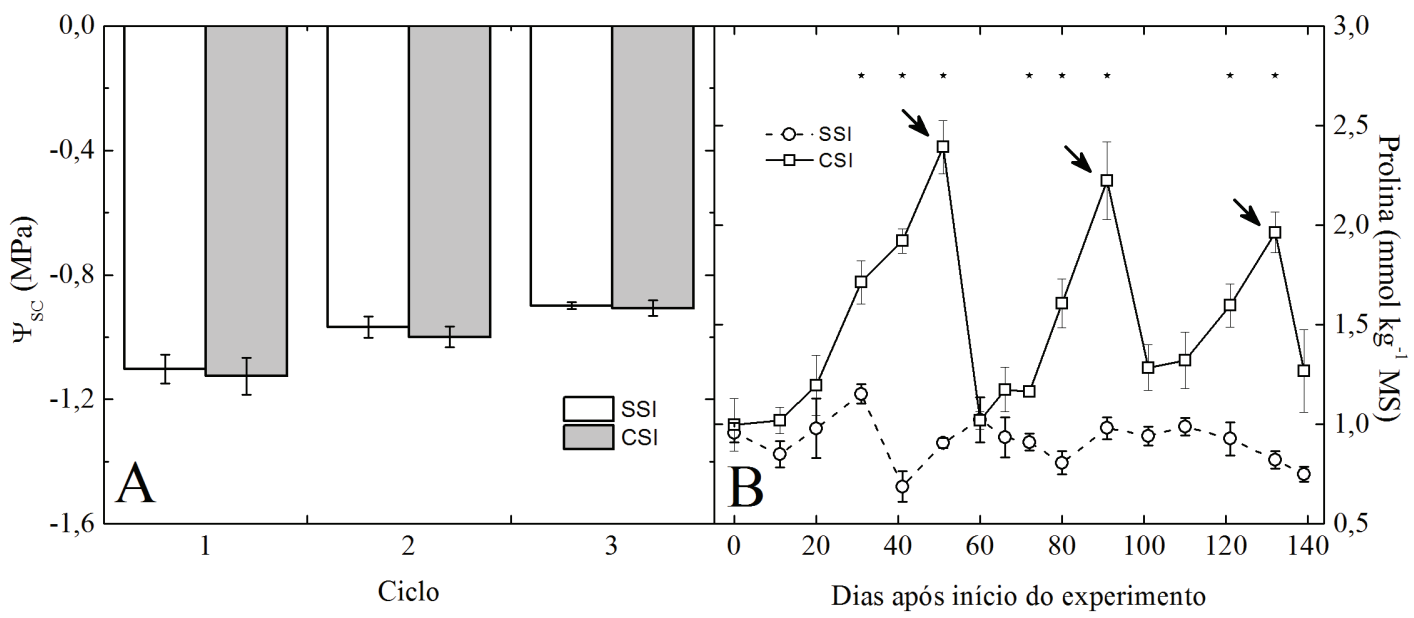

Figura 2. Potencial osmótico corrigido $\left(\Psi_{\mathrm{sC}}\right)$ e concentração de prolina na antemanhã em folíolos de plantas jovens de macaúba. As barras indicam os erros-padrão das médias. O símbolo * indica diferença significativa $(\mathrm{p}<0,05)$, entre as plantas sem $(\mathrm{SSI})$ e com $(\mathrm{CSI})$ suspenção da irrigação. As setas indicam o momento da reirrigação.

O maior acúmulo de prolina nos folíolos ocorreu nos PME, observando-se diferença aos 20 dias antes do PME de cada ciclo S/R (Figura 2B). Com o avanço dos ciclos, houve tendência de redução da concentração de prolina nos picos nos PMEs. O aumento de $143 \%$ nos níveis de prolina na média dos PME dos três ciclos está associado a aspectos bioquímicos e fisiológicos (Roger, 2001). O aumento da síntese e acumulo do prolina em condições de deficiência hídrica tem por objetivo proteger o seu metabolismo em função da desidratação dos tecidos da planta. A prolina, como um osmólito compatível, atua aliviando a acidose citoplasmática, proteção de estruturas subcelulares e macromoléculas, podendo ainda reagir com espécies reativas de oxigênio, inativando-as (Szabados \& Savouré, 2010; Verslues \& Sharma, 2010). A prolina pode atuar como uma reserva de N, a ser suprido à planta após a recuperação de um fator estressante, como por exemplo estresse hídrico (Roger, 2001; Verslues \& Sharma, 2010). Além disso, uma das principais funções da prolina é atuar no ajustamento osmótico em condições de deficiência hídrica, visto que a mesma, por possuir carga elétrica negativa em $\mathrm{pH}$ neutro, não reage de forma a causar dano aos metabólitos celulares. Porém, como não foi observado ajustamento osmótico no presente estudo, supõe-se que o aumento na síntese de prolina atuou de outras formas no metabolismo, exceto no ajustamento osmótico. O aumento de prolina sem ajustamento osmótico significativo também foi observado em ecotipos de $C$. nucifera submetidos à deficiência hídrica (Gomes et al., 2010).

A fluorescência mínima adaptada ao escuro $\left(\mathrm{F}_{0}\right)$ elevou-se nas plantas CSI em relação às SSI aos 41 dias após o início do experimento, e permaneceu com esse comportamento até o final do experimento, exceto na primeira avaliação após a reirrigação do primeiro ciclo, ou seja, durante o período de reidratação das plantas CSI (Figura 3A). Nas plantas SSI os valores de $\mathrm{F}_{0}$ apresentaram média de 160 , ao longo de todo o experimento, e nas plantas $\mathrm{CSI}_{\text {o }} \mathrm{F}_{0}$ chegou a atingir 197. A elevação de $\mathrm{F}_{0}$ nas plantas CSI em relação às SSI sugere um aumento de perda de energia de excitação durante a transferência entre os pigmentos-antena e o centro de reação (Krause \& Weis, 1991). O aumento dos valores de $\mathrm{F}_{0}$ é comum em condições de estresses em plantas, seja ele por frio, salinidade ou déficit hídrico, como no presente estudo, uma vez que a diminuição da transferência de energia de excitação a partir do complexo de antenas colhedoras do fotossintema II para o centro de reação do mesmo evitaria a super excitação do centro de reação (Bergantino et al., 1995; Papageorgiou \& Govindjee, 2004). 

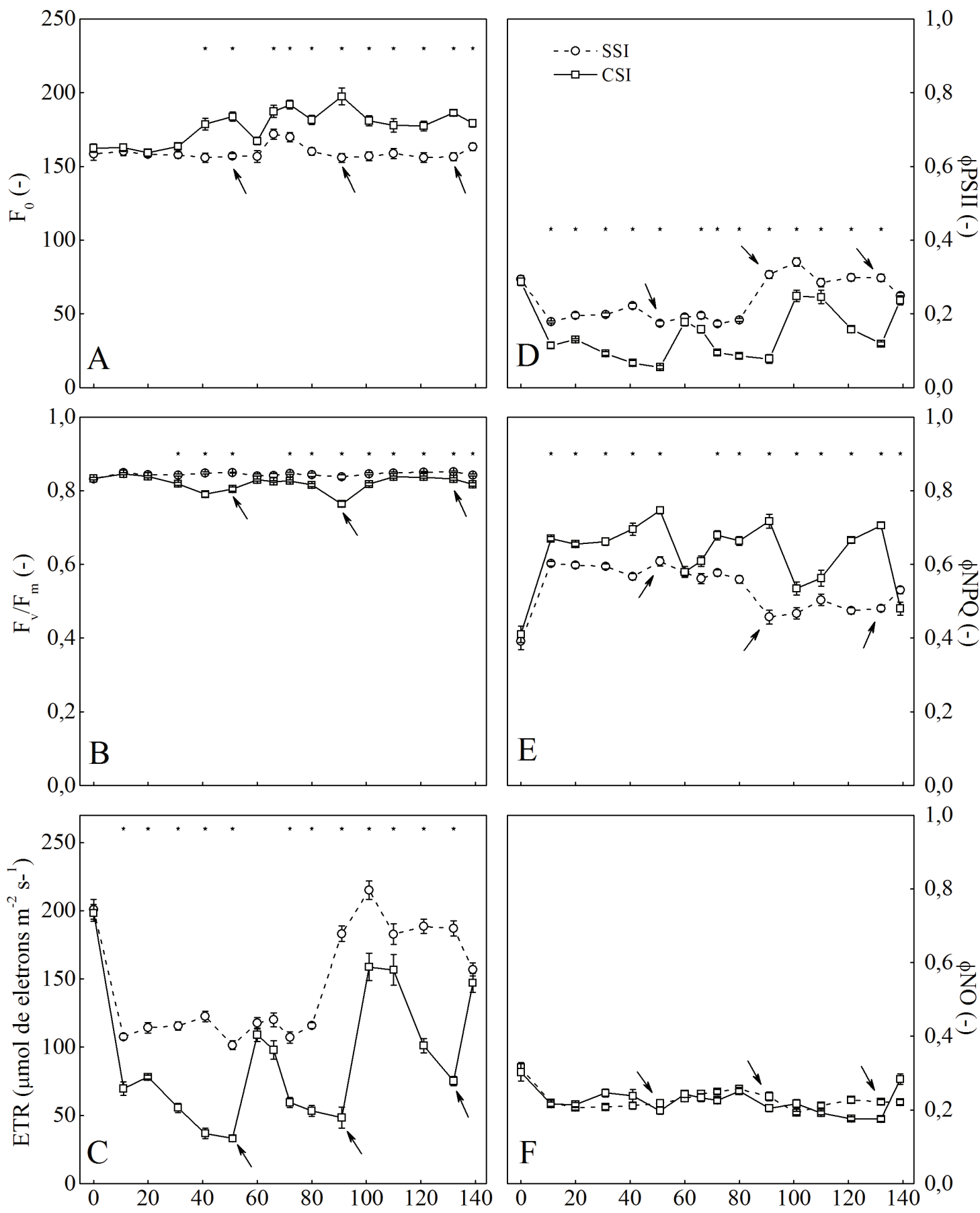

Dias após início do experimento

Figura 3. A) fluorescência mínima $\left.\left(\mathrm{F}_{0}\right), \mathrm{B}\right)$ rendimento quântico máximo do PSII $\left.\left(\mathrm{F}_{\mathrm{v}} / \mathrm{F}_{\mathrm{m}}\right), \mathrm{C}\right)$ taxa aparente de transporte de elétrons (ETR), D) rendimento quântico efetivo do PSII ( $\phi$ PSII), E) rendimento quântico da dissipação regulada de energia não fotoquímica $(\phi N P Q)$ e F) rendimento quântico da dissipação não regulada de energia não fotoquímica no PSII $(\phi N O)$, mensurados em plantas jovens de macaúba. As barras indicam os erros-padrão das médias. O símbolo * indica diferença significativa $(\mathrm{p}<0,05)$, entre as plantas sem (SSI) e com (CSI) suspenção da irrigação. As setas indicam o momento da reirrigação. 
A eficiência quântica máxima do PSII $\left(\mathrm{F}_{\mathrm{v}} / \mathrm{F}_{\mathrm{m}}\right)$ nas plantas SSI, variou de 0,832 a 0,851 , com média de 0,844 durante todo o experimento (Figura 3B). Enquanto nas plantas $\mathrm{CSI}$ a $\mathrm{F}_{\mathrm{v}} / \mathrm{F}_{\mathrm{m}}$, foi reduzida aos 31 dias após o início do experimento, tornando a recupera-se somente nas duas primeiras avaliações do segundo ciclo $\mathrm{S} / \mathrm{R}$. O valor mínimo de $\mathrm{F}_{\mathrm{v}} / \mathrm{F}_{\mathrm{m}}$ observado nas plantas CSI foi de 0,76, indicando possível início de estresse, uma vez que segundo Björkman \& Demmig (1987), o fotodano ocorre abaixo de 0,75 . A redução de $\mathrm{F}_{\mathrm{v}} / \mathrm{F}_{\mathrm{m}}$ pode indicar que teve início o fotodano no aparato fotossintético por produção de espécies reativas de oxigênio (Calatayud et al., 2006), sendo mais expressivo nos PMEs dos ciclos $\mathrm{S} / \mathrm{R} 1$ e 2 , sugerindo que no ciclo 3 as plantas apresentaram certa aclimatação. Uma vez que as plantas podem ter alterado o metabolismo celular para se adaptar às condições de déficit hídrico, com a síntese de prolina (como já discutido anteriormente) e aumento nos níveis de macromoléculas e de enzimas protetoras do estresse oxidativo, evitando assim a oxidação das moléculas de clorofilas (Björkman \& Demmig, 1987; Pintó-Marijuan \& Munné-Bosch, 2014). Reduções significativas de $\mathrm{F}_{\mathrm{v}} /$ $\mathrm{F}_{\mathrm{m}}$ sob deficiência hídrica também foram observadas em E. guineenses (Suresh et al., 2010) e C. nucifera (Gomes et al., 2009), demostrando ser um parâmetro fotossintético importante para a avaliação de fotodano em condições de estresse hídrico.

O rendimento quântico efetivo do PSII ( $\phi$ PSII) foi afetado nas plantas CSI em relação às SSI no decorrer de todo o período, exceto aos zero, 60 e 139 dias após o início do experimento, sendo 60 e 139 dias a recuperação de $A$ referente aos ciclos $\mathrm{S} / \mathrm{R} 1$ e 3 (Figura 3D). Os menores valores de $\phi$ PSII foram observados nos PMEs de cada ciclo $\mathrm{S} / \mathrm{R}$, sendo o menor valor alcançado para $\phi$ PSII de 0,055 . Ao contrário do $\phi$ PSII, o rendimento quântico da dissipação regulada de energia não fotoquímica no PSII ( $\phi N P Q)$, teve elevação ao longo de todos os ciclos $\mathrm{S} / \mathrm{R}$, exceto no dia zero e na recuperação do primeiro ciclo (Figura $3 \mathrm{E}$ ). Os maiores valores de $\phi N P Q$ ocorreram nos PMEs, onde variaram de 0,71 a 0,75 . Em comparação ao $\phi N P Q$ e $\phi P S I I$, que apresentaram elevação ou redução, respectivamente, o rendimento quântico da dissipação não regulada de energia não fotoquímica no PSII ( $\phi \mathrm{NO})$ não apresentou diferença entre as plantas SSI e CSI ao longo do período experimental (Figura 3F). Os valores de $\phi \mathrm{NO}$ variaram de 0,18 a 0,32 , com média de 0,23 .
As alterações no balanço energético nos folíolos de macaúba, promovido pelo déficit hídrico, reduziu o $\phi$ PSII e elevou o $\phi N P Q$, o que representa um redirecionamento da energia absorvida no PSII para a dissipação regulada de energia não fotoquímica do PSII, em vez da utilização desta como energia fotoquímica. Isso demonstra que a energia que era direcionada para a fotoquímica foi reduzida e pode estar ligada à redução de seu uso no ciclo de Calvin (Chaves et al., 2009). Como consequência, o $\phi \mathrm{NPQ}$ apresentou incrementos, o que é indicativo de uma capacidade fotoprotetora do aparato fotossintético (Baraldi et al., 2008; Moustaka \& Moustakas, 2014), a qual é observada com a recuperação de $A$ após a reidratação.

A participação do $\phi N P Q$ na dissipação da energia total absorvida envolve a dissipação na forma de calor pelo ciclo das xantofilas (Baraldi et al., 2008; Moustaka \& Moustakas, 2014), pois a protonação do lúmen do tilacoide ativa esse ciclo, acarretando desepoxidação da violaxantina, que formará a anteraxantina, e esta por sua vez formará a zeaxantina (Baraldi et al., 2008). A capacidade de dissipação de energia e proteção metabólica contra os efeitos danosos das espécies reativas de oxigênio, gerados pela transferência de elétrons ao $\mathrm{O}_{2}$ no FSI ou através da reação de Mehler, pode constituir-se numa característica-chave para a sobrevivência das plantas em condições de seca (Chaves et al., 2009). A estabilidade de $\phi \mathrm{NO}$ ao longo dos ciclos $\mathrm{S} / \mathrm{R}$, indica que os mecanismos de regulação de proteção do PSII é eficiente em macaúba, e não falharam sob deficiência hídrica.

Como resultado da redução de $\phi$ PSII e aumento do $\phi N P Q$, a taxa aparente de transporte de elétrons (ETR) foi reduzida logo após a suspenção da irrigação, tendo retornado a valores similares às plantas SSI somente durante a rehidratação dos ciclos 1 e 3 (Figura 3C). A maior redução de ETR em cada ciclo ocorreu no PME, igualmente ao $\phi$ PSII, algo esperado, visto que o $\phi$ PSII influência diretamente a ETR. A ETR aumentou com o avanço dos ciclos $\mathrm{S} / \mathrm{R}$, apresentando valores de 33,2, 48,4 e 75,2 $\mu \mathrm{mol}$ de elétrons $\mathrm{m}^{-2} \mathrm{~s}^{-1}$, para os ciclos 1,2 e 3, respectivamente. Essa menor redução dos valores de ETR, bem como os de $\phi$ PSII e aumento de $\phi$ NPQ e inalteração de $\phi \mathrm{NO}$, com o avanço dos ciclos, denota uma possível aclimatação das plantas de macaúba à deficiência hídrica. A manutenção dos valores de ETR acima de 33,2 $\mu \mathrm{mol}$ de elétrons $\mathrm{m}^{-2} \mathrm{~s}^{-1}$ nos PME, ou 
seja, quando $A$ era muito próximo a zero, demostra que o funcionamento do ciclo água-água e a fotorrespiração em condições de deficiência hídrica auxiliaram na manutenção da ETR (Moustaka \& Moustakas, 2014; Noctor et al., 2002). Essa manutenção de ETR nos PMEs indica que, apesar de haver limitação não estomática da fotossíntese, o transporte de elétrons está ativo (Moustaka \& Moustakas, 2014).

A suspensão da irrigação em plantas jovens de macaúba não afetou o conteúdo de carotenoides e de clorofilas totais (Figura 4). A constância entre os valores de clorofilas e carotenoides ao longo dos ciclos $\mathrm{S} / \mathrm{R}$, assim como os valores de $\phi \mathrm{NO}$ onde esse representa a perda de energia constitutiva, demonstra que a macaúba possui um efetivo sistema de dissipação de energia representado pelo $\phi$ PSII e $\phi N P Q$. Sistema esse composto pela fotorrespiração, ciclo água-água e das xantofilas, além da própria fotossíntese, que atuam em conjunto na fotoproteção do aparato fotossintético e pode ser categorizada em processos fotoquímicos ou dissipação térmica, sendo esse último proveniente tanto dos complexos de colheita de luz, como dos centros de reação do PSII (Calatayud et al., 2006; Hendrickson et al., 2004; Moustaka \& Moustakas, 2014).

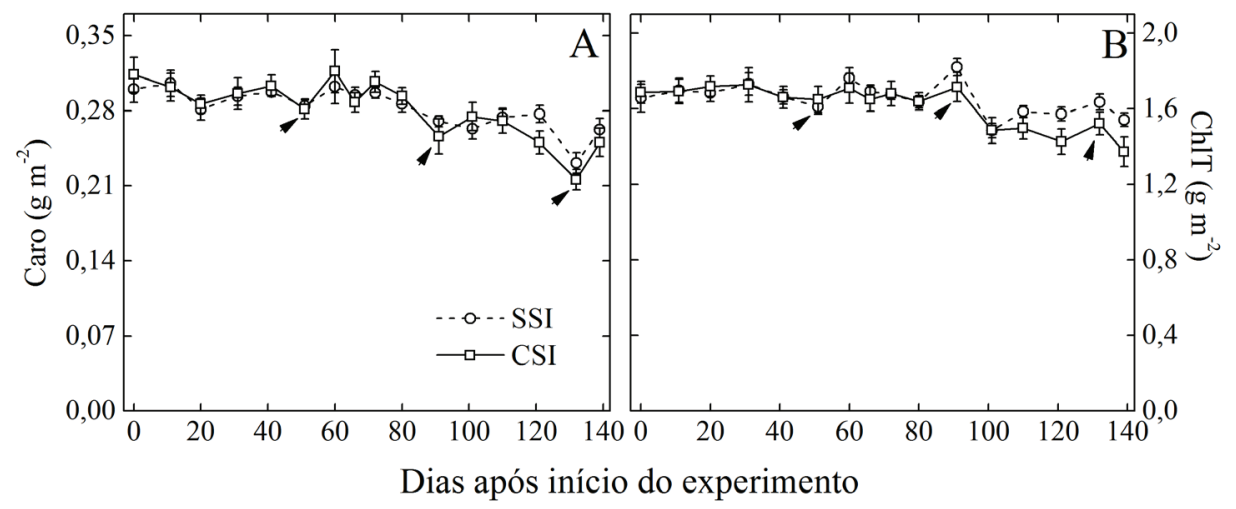

Figura 4. A) Concentração de carotenoides (Car) e B) teor de clorofilas totais (ChlT) em folíolos de plantas jovens de macaúba. As barras indicam os erros-padrão das médias. $\mathrm{O}$ símbolo * indica diferença significativa $(\mathrm{p}<0,05)$, entre as plantas sem $(\mathrm{SSI})$ e com $(\mathrm{CSI})$ suspenção de irrigação. As setas indicam o momento da reirrigação.

\section{Conclusões}

Há indicativo da aclimatação da macaúba em condições de deficiência hídrica, com o avanço dos ciclos seca/reirrigação.

A macaúba não é efetiva em promover ajustamento osmótico sob deficiência hídrica, apesar do acúmulo de prolina, mas apresenta eficiente sistema de dissipação regulada de energia não fotoquímica no PSII ( $\phi N P Q)$.

$O$ balanço de energia, representado por $\phi$ PSII, $\phi N P Q$ e $\phi N O$, é efetivo para o aproveitamento e dissipação de energia, de forma a não afetar a fluorescência constitutiva.

\section{Agradecimentos}

A Fapemig pelo auxílio financeiro parcial para execução do projeto.

\section{Referências}

Alves, A. A. C. \& Setter, T. L. Abscisic acid accumulation and osmotic adjustment in cassava under water deficit. Environmental and Experimental Botany, v. 51, n. 3, p. 259-271, 2004. DOI: 10.1016/j.envexpbot.2003.11.005.

Baer, R. J. \& Baldwin, K. A. Freezing points of bulking agents used in manufacture of low-calorie frozen desserts. Journal of Dairy Science, v. 67, n. 12, p. 2860-2862, 1984. DOI: 10.3168/jds.S00220302(84)81647-1.

Baraldi, R. et al. Role of xanthophyll cycle-meiated photoprotrection in Arbutus unedo plants exposed to water stress during the Mediterranean summer. Photosynthetica, v. 46, n. 3, p. 378-386, 2008. DOI: 10.1007/s11099-008-0069-x.

Bates, L. et al. Rapid determination of free proline for water stress studies. Plant and Soil, v. 39, n. 1, p. 205-207, 1973. DOI: 10.1007/ BF00018060.

Bergantino, E. et al. A post-translational modification of the photosystem II subunit CP29 protects maize from cold stress. The Journal of Biological Chemistry, v. 270, n. 15, p. 8474-8481, 1995. 
Björkman, O. \& Demmig, B. Photon yield of O2 evolution and chlorophyll fluorescence characteristics at $77 \mathrm{~K}$ among vascular plants of diverse origins. Planta, v. 170, n. 4, p. 489-504, 1987. DOI: $10.1007 / \mathrm{BF} 00402983$.

Calatayud, A. et al. Spatial-temporal variations in rose leaves under water stress conditions studied by chlorophyll fluorescence imaging. Plant Physiology and Biochemistry, v. 44, n. 10, p. 564-573, 2006. DOI: 10.1016/j.plaphy.2006.09.015.

Calbo, M. E. R. \& Moraes, J. A. P. V. Efeitos da deficiência hídrica em plantas de Euterpe oleracea (açaí). Revista Brasileira de Botânica, v. 23, n. 3, p. 225-230, 2000. DOI: 10.1590/S010084042000000300001 .

Chaves, M. M. et al. Photosynthesis under drought and salt stress: regulation mechanisms from whole plant to cell. Annals of Botany, v. 103 , n. 4 , p. $551-560,2009$. DOI: $10.1093 / \mathrm{aob} / \mathrm{mcn} 125$.

Cha-Um, S. et al. Physio-biochemical responses of oil palm (Elaeis guineensis Jacq.) seedlings to mannitol- and polyethylene glycolinducediso-osmotic stresses. Plant Production Science, v. 15, n. 2, p. $65-72,2012$. DOI: $10.1626 /$ pps. 15.65 .

Claeys, H. \& Inzé, D. The agony of choice: how plants balance growth and survival under water-limiting conditions. Plant Physiology, v. 162, n. 4, p. 1768-1779, 2013. DOI: 10.1104/pp.113.220921.

Cornic, G. Drought stress inhibits photosynthesis by decreasing stomatal aperture - not by affecting ATP synthesis. Trends in Plant Science, v. 5, n. 5, p. 187-188, 2000. DOI: 10.1016/S13601385(00)01625-3.

Dourado-Neto, D. et al. Software to model soil water retention curves (SWRC, version 2.00). Scientia Agricola, v. 57, n. 1, p. 191-192, 2000. DOI: 10.1590/S0103-90162000000100031.

Flexas, J. et al. Keeping a positive carbono balance under adverse conditions: responses of photosynthesis and respiration to water stress. Physiologia Plantarum, v. 127, n. 3, p. 343-352, 2006. DOI: 10.1111/j.1399-3054.2006.00621.x

Gomes, F. P. et al. Is abscisic acid involved in the drought reponses of brazilian green dwarf coconut? Experimental Agriculture, v. 45, n. 2, p. 189-198, 2009. DOI: 10.1017/S0014479708007436.

Gomes, F. P. et al. Osmotic adjustment, proline accumulation and cell membrane stability in leave of Cocos nucifera submitted to drought stress. Scientia Horticulturae, v. 126, n. 3, p. 379-384, 2010. DOI: 10.1016/j.scienta.2010.07.036.

Gomes, F. P. et al. Photosynthetic limitations in leaves of young brazilian green dwarf coconut (Cocos nucifera L. 'nana') palm under well-watered conditions or recovering from drought stress. Environmental and Experimental Botany, v. 62, n. 3, p. 195-204, 2008. DOI: 10.1016/j.envexpbot.2007.08.006.

Hendrickson, L. et al. A simple alternative approach to assessing the fate of absorbed light energy using chlorophyll fluorescence. Photosynthesis Research, v. 82, n. 1, p. 73-81, 2004. DOI: 10.1023/B:PRES.0000040446.87305.f4.

Henriques, G. S. \& Rosado, G. P. Formulação de dietas enterais artesanais e determinação da osmolalidade pelo método crioscópico. Revista de Nutrição, v. 12, n. 3, p. 225-232, 1999. DOI: 10.1590/ S1415-52731999000300003.
Krasensky, J. \& Jonak, C. Drought, salt, and temperature stressinduced metabolic rearrangements and regulatory networks. Journal of Experimental Botany, v. 63, n. 4, p. 1593-1608, 2012. DOI: 10.1093/jxb/err460.

Krause, G. H. \& Weis, E. Chlorophyll fluorescence and photosynthesis: the basics. Annual Review of Plant physiology and Plant Molecular Biology, v. 42, p. 313-349, 1991. DOI: 10.1146/annurev. pp.42.060191.001525.

Lipiec, J. et al. Effect of drought and heat stresses on plant growth and yield: a review. International Agrophysics, v. 27, n. 4, p. 463477, 2013. DOI: 10.2478/intag-2013-0017.

Moustaka, J. \& Moustakas, M. Photoprotective mechanism of the non-target organism Arabidopsis thaliana to paraquat exposure. Pesticide Biochemistry and Physiology, v. 111, p. 1-6, 2014. DOI: 10.1016/j.pestbp.2014.04.006.

Noctor, G. et al. Drought and oxidative load in the leaves of C3 plants: a predominant role for photorespiration? Annals of Botany, v. 89, n. 7, p. 841-850, 2002. DOI: 10.1093/aob/mcf096.

Papageorgiou, G. C. \& Govindjee. Chlorophyll a fluorescence: a signature of photosynthesis. Dordrech: Springer, 2004. 820 p.

Pimentel, L. D. et al. Recomendação de adubação e calagem para o cultivo da macaúba: 1a aproximação. Informe Agropecuário, v. 32, n. 265, p. 20-31, 2011.

Pintó-Marijuan, M. \& Munné-Bosch, S. Photo-oxidative stress markers as a measure of abiotic stress-induced leaf senescence: advantages and limitations. Journal of Experimental Botany, v. 64, n. 14, p. 3845-3857, 2014. DOI: 10.1093/jxb/eru086.

Richards, L. A. Porous plate apparatus for measuring moisture retention and transmission by soils. Soil Science, v. 66, n. 2, p. 105-110, 1948. DOI: 10.1097/00010694-194808000-00003

Roger, M. R. Handbook of plant ecophysiology techniques. Dordrecht: Kluwer Academic Publishers, 2001. 452 p.

SAS Institute Inc. Statistical analysis system user's guide: version 9.1. Cary, 2004. 5136 p.

Suresh, K. et al. Changes in photosynthetic activity in five common hybrids of oil palm (Elaeis guineensis Jacq.) seedlings under water deficit. Photosynthetica, v. 50, n. 4, p. 549-556, 2012. DOI: 10.1007/ s11099-012-0062-2.

Suresh, K. et al. Gas-exchange characteristics, leaf water potential and chlorophyll a fluorescence in oil palm (Elaeis guineensis Jacq.) seedlings under water stress and recovery. Photosynthetica, v. 48, n. 3, p. 430-436, 2010. DOI: 10.1007/s11099-010-0056-x.

Szabados, L. \& Savouré, A. Proline: a multifunctional amino acid. Trends in Plant Science, v. 15, n. 2, p. 89-97, 2010. DOI: 10.1016/j. tplants.2009.11.009.

Verslues, P. E. \& Sharma, S. Proline Metabolism and Its Implications for Plant-Environment Interaction. The Arabidopsis Book, v. 8, p. 1-23, 2010. DOI: 10.1199/tab.0140.

Wellburn, A. R. The spectral determination of chlorophylls a and $\mathrm{b}$, as well as total carotenoids, using various solvents with spectrophotometers of different resolution. Journal of Plant Physiology, v. 144, n. 3, p. 307-313, 1994. DOI: 10.1016/S01761617(11)81192-2. 